\title{
The INES system
}

\section{Ripple correction and absolute calibration for IUE high resolution spectra}

\author{
A. Cassatella ${ }^{1,2, \star}$, A. Altamore ${ }^{2}$, R. González-Riestra ${ }^{3, \star}$, J.D. Ponz ${ }^{4}$, J. Barbero ${ }^{5}$, A. Talavera ${ }^{3, \star}$, and \\ W. Wamsteker ${ }^{6, \star \star}$ \\ ${ }^{1}$ CNR, Istituto di Astrofisica Spaziale, Via del Fosso del Cavaliere, I-00133 Roma, Italia \\ 2 Dipartimento di Fisica E. Amaldi, Universitá Roma Tre, Via della Vasca Navale 84, I-00146 Roma, Italia \\ 3 Laboratorio de Astrofísica Espacial y Física Fundamental, VILSPA, P.O. Box 50727, E-28080 Madrid, Spain \\ 4 European Space Agency, VILSPA, P.O. Box 50727, E-28080 Madrid, Spain \\ 5 Departamento de Física Aplicada, Universidad de Almería, Carretera de Sacramento s/n, E-04120 Almería, Spain \\ ${ }^{6}$ ESA-IUE Observatory, VILSPA, P.O. Box 50727, E-28080 Madrid, Spain
}

Received July 23; accepted October 22, 1999

\begin{abstract}
In this paper we document the results of the study which led to the ripple correction and absolute calibration algorithms applied to the high resolution spectra processed with the NEWSIPS software for the Final Archive of the IUE Project. In this analysis, based on a very large number of spectra, we find that both the $K$ and $\alpha$ parameters (not only the former as previously believed) vary with order number. This fact, together with the finding that the central peaks of the blaze function vary also as a function of the THDA temperature (for the SWP camera) and of the date of observations (for the LWP and LWR cameras), makes the ripple correction algorithm more complex than previously considered but, at the same time, considerably more reliable. As for the high resolution absolute calibration, the method followed is similar to the one implemented in IUESIPS. The internal accuracy of the high resolution calibration is about $4 \%$. We note that the ripple correction and absolute calibration algorithms here described apply also to IUE data processed and distributed with the INES system.
\end{abstract}

Key words: methods: data analysis; techniques: image processing; ultraviolet: general

\section{Introduction}

In this paper we address two fundamental aspects which influence, to different extent, the photometric quality of

Send offprint requests to: A. Cassatella,

e-mail: cassatella@fis.uniroma3.it

* Previously: ESA-IUE Observatory.

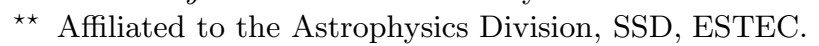

$I U E$ high resolution spectra, i.e. the correction for the echelle blaze function (ripple correction), and the absolute calibration. The results of this study, which are based on about one thousand spectra, mainly of $I U E$ standard stars, have been implemented in the IUE Newly Extracted Spectra system (INES), a data archive and distribution facility developed by the IUE ESA project (see Wamsteker et al. 1999), as well as in the NEWSIPS data reduction package (Nichols \& Linsky 1996; Garhart et al. 1997). Full details on the INES system are provided by RodríguezPascual et al. (1999, Paper I) for low resolution data, and by González-Riestra et al. (1999a, Paper III) for high resolution data.

\section{The NEWSIPS ripple correction}

The correction of $I U E$ high resolution spectra for the echelle grating efficiency has for long been considered a critical area in the context of IUE data reduction procedures. The INES system includes an upgraded ripple correction algorithm which is presented and discussed in the following.

\subsection{The method}

Let us indicate with $R_{m}(\lambda)$ the grating efficiency (blaze function) of the high resolution spectrographs, for a given order $m$ and wavelength $\lambda$. As shown by Ahmad (1981) and Ake (1981), this function is adequately represented by:

$R_{m}(\lambda)=\sin ^{2} x / x^{2}$ 
where

$x=\pi m \alpha\left(1-\lambda_{\mathrm{c}}(m) / \lambda\right)$

being $\lambda_{c}(m)$ the wavelength of the central maximum of the blaze function for order $m$, and $\alpha$ a parameter which can be shown to be, in the first approximation, inversely proportional to the Half Width at Half Maximum (HWHM) of the order considered:

$\mathrm{HWHM}=1.395 \lambda_{\mathrm{c}}(m) /(\pi m \alpha)$.

The quantity

$K=m \lambda_{\mathrm{c}}(m)$

is called the "ripple constant", but, as discussed in the following, it turns out to be a function of order number for the $I U E$ setup.

Let us indicate with $f(\lambda)$ the observed net spectrum normalized to the exposure time. The shape of the blaze function $R_{m}(\lambda)$ can be determined from the observations, knowing the inverse sensitivity curve of the camera $\left(S^{-1}(\lambda) C(\lambda)\right.$, see Sect. 3), and the absolute flux of the target $F(\lambda)$ :

$R_{m}(\lambda)=f(\lambda) C(\lambda) S^{-1}(\lambda) / F(\lambda)$.

This equation implies that the blaze parameters cannot be derived from $f(\lambda)$ directly, because it is affected by the distortions introduced by the multiplying factor $C(\lambda) S^{-1}(\lambda) / F(\lambda)$, which is not only wavelengthdependent, but differs also from target to target. Not having taken this fact into account is one of the major causes for the inaccuracy of the ripple correction in previous releases of the $I U E$ data reduction packages and, in particular, for the suspected dependence of the ripple parameters on the spectral type of the source considered.

Our approach to derive the ripple parameters is equivalent to using Eq. (5), but is of much easier implementation. It consists in normalizing the observed spectrum to its continuum. The continuum was determined, for each spectral order, in correspondence to the expected peaks of the blaze function (Grady \& Garhart 1989), where $R_{m}(\lambda) \simeq 1$, and then interpolated over wavelength.

Let us indicate with $Y(\lambda)$ the net spectrum extracted from order $m$ after normalization to the continuum, and with $T(m, \lambda)$ its analytical representation. We can write:

$T(m, \lambda)=A_{m} R_{m}(\lambda)$

where $A_{m}$ is an adjustable parameter representing the net peak intensity of the blaze function (at $\lambda=\lambda_{\mathrm{c}}$ ). Because of normalization to the continuum, $A_{m} \simeq 1$. The unknown blaze parameters $\alpha, \lambda_{\mathrm{c}}(m)$ and $A_{m}$ were determined iteratively, for each order, via a non-linear least-squares fitting algorithm, i.e. by minimizing $\sum\left(T_{i}-Y_{i}\right)^{2}$. All the data points were given the same weight except those affected by saturation, ITF extrapolation, reseau marks or particle events, which were discarded. Spectral absorption features were also discarded through an automatic rejection
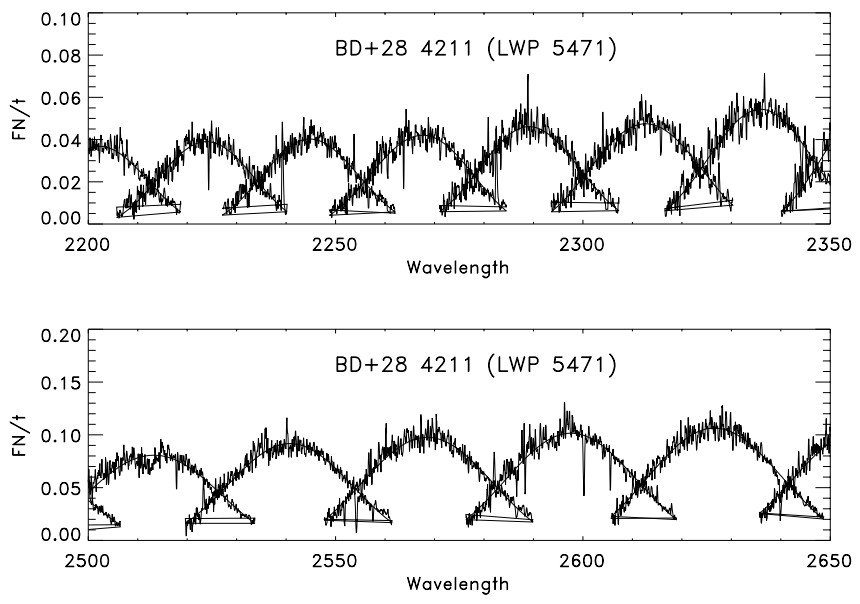

Fig. 1. Example of fitting Eq. (1) to a portion of a LWP spectrum of $\mathrm{BD}+284211$

procedure. An example of the fitting for the LWP05471 spectrum of the standard star BD+28 4211 is shown in Fig. 1.

We stress that the ripple parameters should be derived, as done here, in vacuum wavelengths and in the velocity scale of the $I U E$ spacecraft.

\subsection{The data base}

For this study we have selected 516 good quality high resolution spectra of the IUE calibration standards obtained between 1978 and 1995, and namely: 182 spectra for the SWP camera, 296 for the LWP and 138 for the LWR. This represents the near totality of data of this kind available. As input data we used the MHXI net spectra as extracted with NEWSIPS. Among the most frequently observed standards we quote $\mathrm{BD}+28$ 4211, BD+75 325, HD 60753, HD 3360, G191 B2B, NGC 246, HD 120315 and HD 93521. In addition, to test specific aspects of the ripple correction algorithm, it was necessary to introduce about 200 net spectra (also extracted with NEWSIPS) of targets having heterogeneous spectral types. The large majority of the spectra were obtained through the large entrance apertures of the $I U E$ spectrographs. A few small aperture spectra were also considered, whenever available, to cross-check the applicability of the large aperture ripple correction algorithm to that case. For the long wavelength cameras, several overexposed spectra were also included to refine the ripple parameters at the shortest wavelengths, where the cameras sensitivity is low.

\subsubsection{SWP}

Dependence of $K$ on order number: We find that the central positions of spectral orders $\lambda_{\mathrm{c}}(m)$ are not stable for SWP spectra, as commonly believed in the past 
but, for a given order, vary strongly as a function of the Camera Head Temperature (THDA). Given this, a convenient way to proceed is to determine first $\lambda_{c}(m)$ from data obtained within a restricted range of THDA values. We used 52 spectra of the stars BD $+28^{\circ} 4211$ (13 spectra), $\mathrm{BD}+75^{\circ} 325$ (20 spectra), CD-38²10980 (5 spectra), HD 60753 (12 spectra) and NGC 246 (2 spectra) obtained the range $8.5 \leq T H D A \leq 11.5^{\circ} \mathrm{C}$. Being the mean value of THDA for this sample, $10.04 \pm 0.70^{\circ} \mathrm{C}$, very close to the average operating conditions for the SWP camera during years 1978 to 1991, the corresponding central positions $\lambda_{c}(m)$ can be used to obtain a mean curve $K=m \lambda_{\text {ref }}$, where $\lambda_{\text {ref }}=\lambda_{c}(m$, THDA $=10.04)$ are the central wavelengths to be taken as a reference for that temperature. A linear regression to the mean values for the four stars provides:

$K=m \lambda_{\text {ref }}=137508.316+2.44761341 m$.

The associated uncertainty on the central wavelengths is $0.24 \AA$ at $1400 \AA$ and $0.31 \AA$ at $1850 \AA$. The ripplecorrected fluxes in the overlap region between adjacent orders are accurate to within $3 \%$ above $1700 \AA$ and $6 \%$ around $1200 \AA$. However, the flux mismatch in the overlap region between adjacent orders caused by a systematic error on the central wavelengths is twice the quoted errors, because if the flux at order $\mathrm{m}$ is overestimated, the flux in the overlap region of the adjacent order $m-1$ will be underestimated and vice versa.

Dependence of $\lambda_{\mathrm{c}}$ on THDA: To extend the validity of Eq. (7) to any regime of THDA, we have used the whole set of input spectra and computed for each spectrum the wavelength difference between the observed central wavelengths and those obtained from Eq. (7). A linear fit to the data over the range of orders $110-86$, where the blaze function is narrower, and then better sampled all over its shape, provides:

$\lambda_{\mathrm{c}}-\lambda_{\text {ref }}=-0.335775+0.0321729$ THDA

with a standard deviation of $0.069 \AA$. This equation quantifies the previously quoted dependence of the central positions on THDA. We have also looked for a possible dependence of the central wavelengths on the epoch of the observation, on the spectral type of the target and on the exposure level, but no correlation was found.

Dependence of $\alpha$ on order number and focussing conditions: We find that the ripple parameter $\alpha$ varies linearly as a function of the order number. A linear fit to the data provides:

$\alpha=0.92628-7.89013210^{4} \mathrm{~m}$
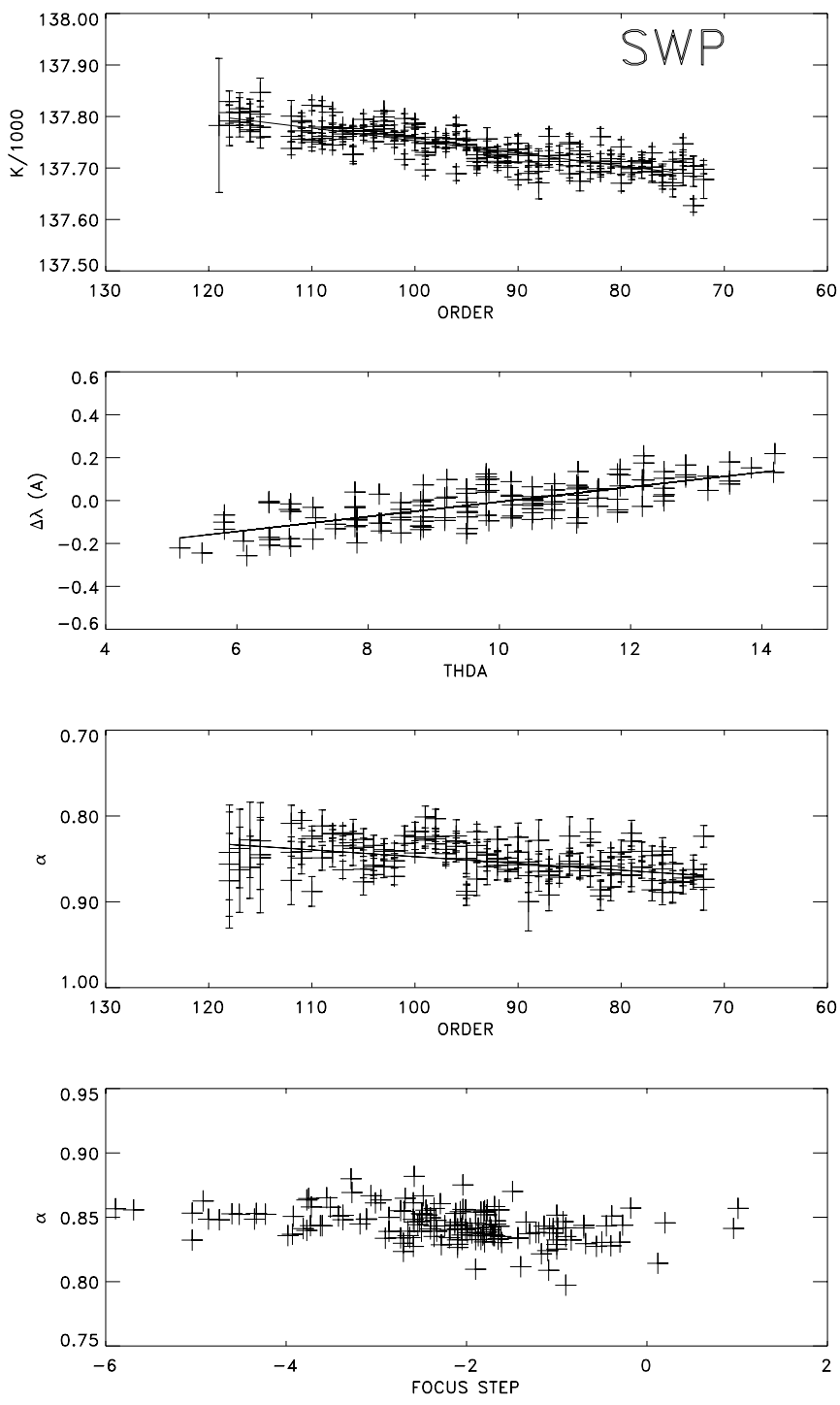

Fig. 2. Ripple parameters for the SWP camera. Form top to bottom: Variation of $K$ with order number, dependence of $\Delta \lambda$ on THDA, and dependence of $\alpha$ on order number and on focus STEP

with a standard deviation of 0.022 . The corresponding uncertainty on fluxes in the regions midway between adjacent orders is $3.6 \%$, irrespective of the order considered. Note that the errors in $\alpha$ do not produce any flux discontinuity in the overlap region between adjacent orders.

No dependence of $\alpha$ on the date of observation or THDA was detected. On the contrary, there is a marginal indication of a slight decrease of $\alpha$ with increasing focus STEP parameter. This effect, if real, would imply that the width of the spectral orders becomes larger as STEP departs from the optimum focussing conditions. Since most IUE data are taken at optimum focussing conditions and the effect is any case marginal, it has not been implemented in the ripple correction algorithm. In Fig. 2 we show the ripple constant $\mathrm{K}$ as a function of order number, 


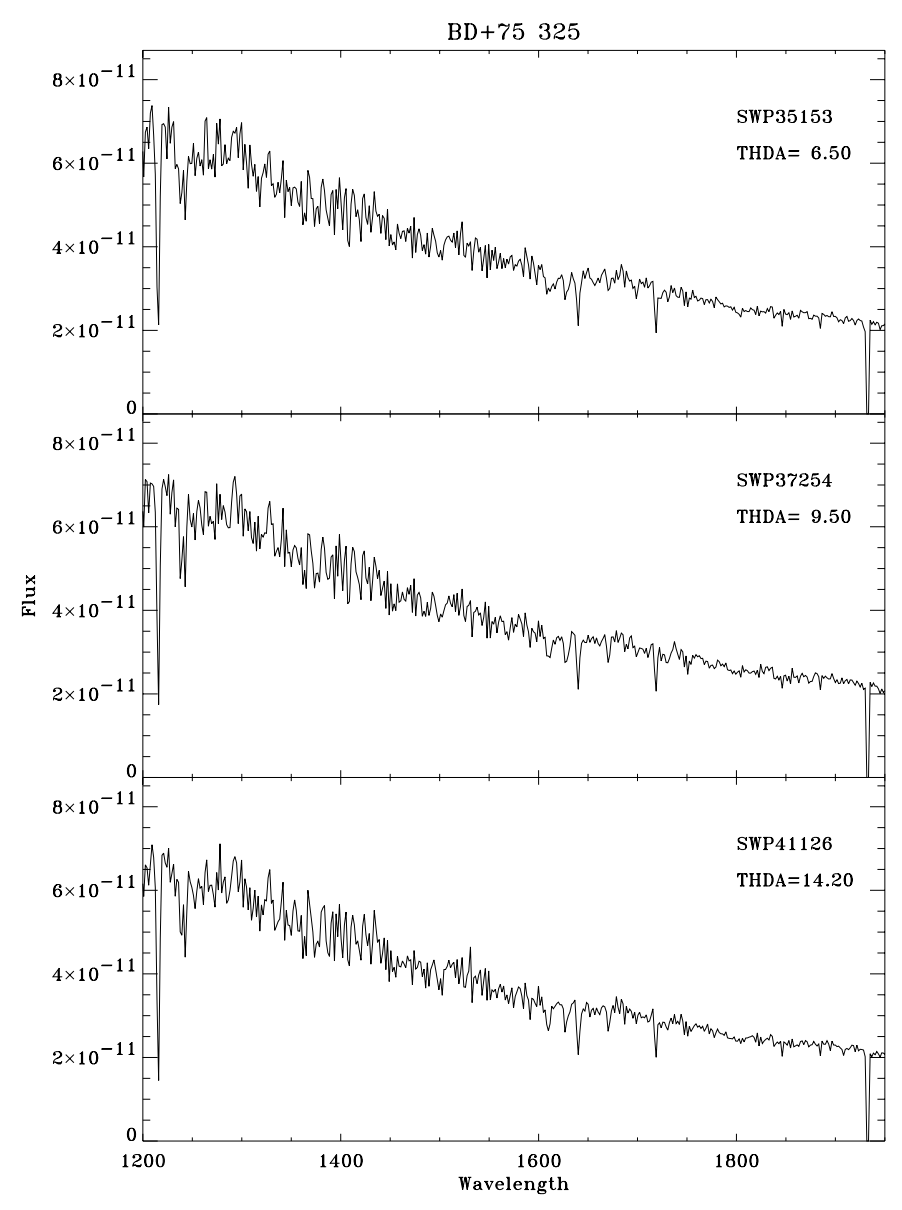

Fig. 3. Energy distribution of BD+75 325 obtained from three SWP ripple corrected high resolution rebinned spectra obtained under very different THDA conditions. Fluxes are in units of erg cm $\mathrm{cm}^{-2} \mathrm{~s}^{-1} \AA^{-1}$

the wavelength shift $\Delta \lambda=\lambda_{\mathrm{c}}-\lambda_{\text {ref }}$ as a function of THDA, and the $\alpha$ parameter as a function of order number and focus STEP.

Given the strong dependence of the central wavelengths of spectral orders on the THDA temperature, we have verified that the SWP ripple correction algorithm works well even when the THDA values depart considerably from the average operating conditions. As an example, we show in Fig. 3, three spectra of BD+75 325 obtained with very different THDA values (6.5, 9.5 and 14.2): no periodic flux fluctuations (typical of bad ripple correction) are seen in the corrected spectra.

\subsubsection{LWP}

Dependence of $K$ on order number: Contrary to the case of the SWP camera, the central wavelengths of LWP spectral orders $\lambda_{\mathrm{c}}(m)$ are not sensitive to the camera head temperature THDA, but depend strongly on the date of the observations.
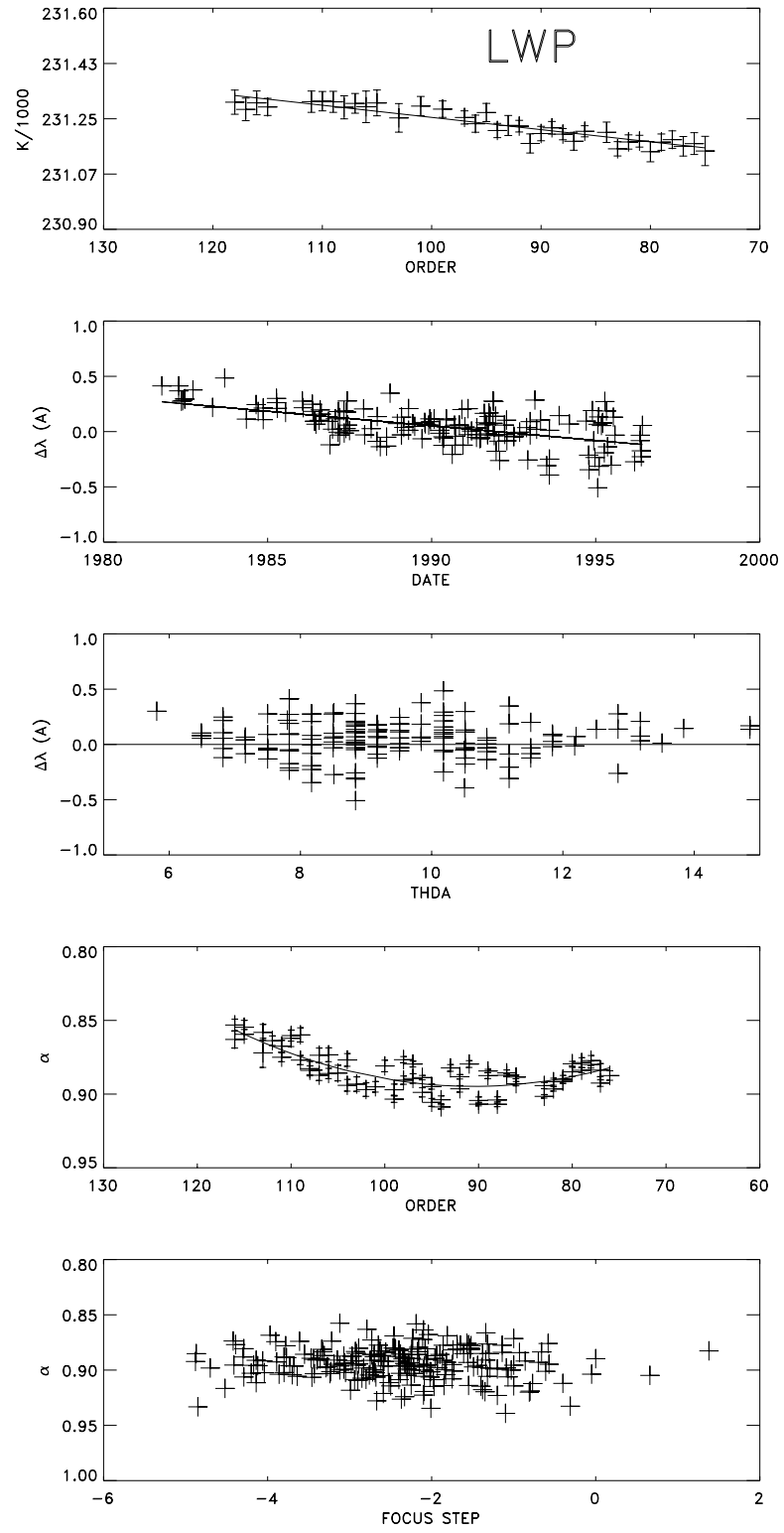

Fig. 4. Ripple parameters for the LWP camera. Form top to bottom: the $K$ parameter as a function of order number; the wavelength shift $\Delta \lambda=\lambda_{\mathrm{c}}-\lambda_{\text {ref }}$ as a function of observing date and THDA; the $\alpha$ parameter as a function of order number and focus STEP. Note the lack of correlation between $\Delta \lambda$ and THDA, and between $\alpha$ and focus STEP

It is then convenient to study first the dependence of $K$ on order number for spectra obtained in a sufficiently short time interval. We have selected the period from year 1988 to 1992 because many (86) good quality spectra of the IUE calibration standards were obtained. The mean observing date of this restricted sample was $T=1991.2$ (fractional year), and the mean and rms values of THDA and focus STEP were $10.18 \pm 1.81^{\circ} \mathrm{C}$ and $-2.84 \pm 0.85$, respectively. Since the central positions were sufficiently stable in the quoted period of time, the values 


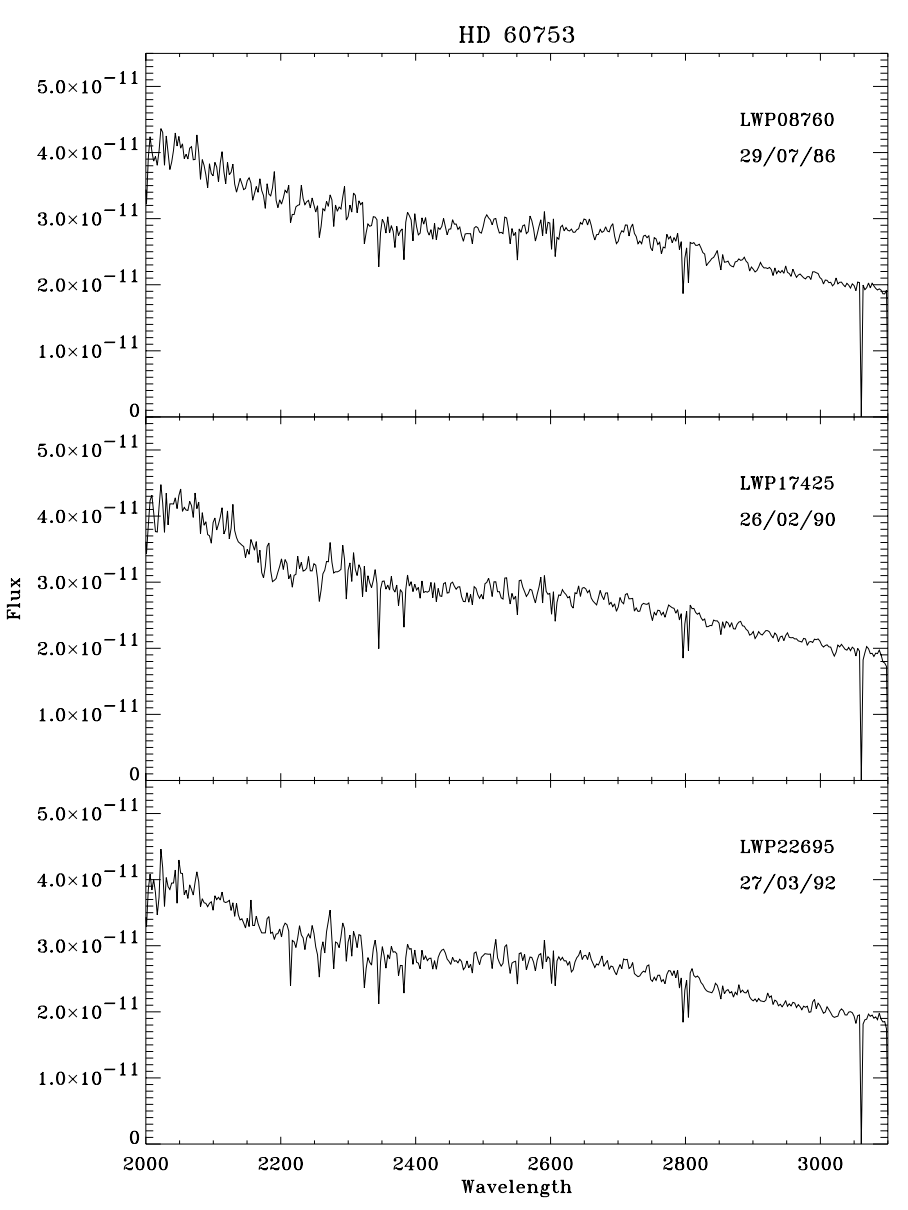

Fig. 5. Flux distribution of HD 3360 obtained from three LWP ripple corrected and flux calibrated high resolution rebinned spectra obtained at very different epochs. Fluxes are in the same units as in Fig. 3

of $K=m \lambda_{\mathrm{c}}(m, T=1991.2)$ obtained from the individual spectra were averaged together. A linear regression to these data provides:

$K=m \lambda_{\text {ref }}(m)=230868.17770+3.86260914 m$

where $\lambda_{\text {ref }}=\lambda_{\mathrm{c}}(m, T=1991.2)$ are the reference central wavelengths for time $T=1991.2$. The standard deviation of the fit is 30.1 , corresponding to an uncertainty on the central wavelengths of $0.30 \AA$ at $2300 \AA$. The ripple corrected fluxes in a point midway in the overlap region between adjacent orders are accurate to within $3.9 \%$ at $2200 \AA$, and $2.3 \%$ at $2800 \AA$.

Dependence of $\lambda_{\mathrm{c}}$ on time: In this section we show that the central wavelengths $\lambda_{\mathrm{c}}$ of a given order vary with time, being their deviations with respect to the mean values in 1991.2 (see Eq. 10) a linear function of observing time. To define this dependence, we have selected 142 spectra obtained between 1981 and 1995, and derived, for each order, the wavelength difference with respect to the reference values for 1991.2. Since the shape of the $K(m)$ func-
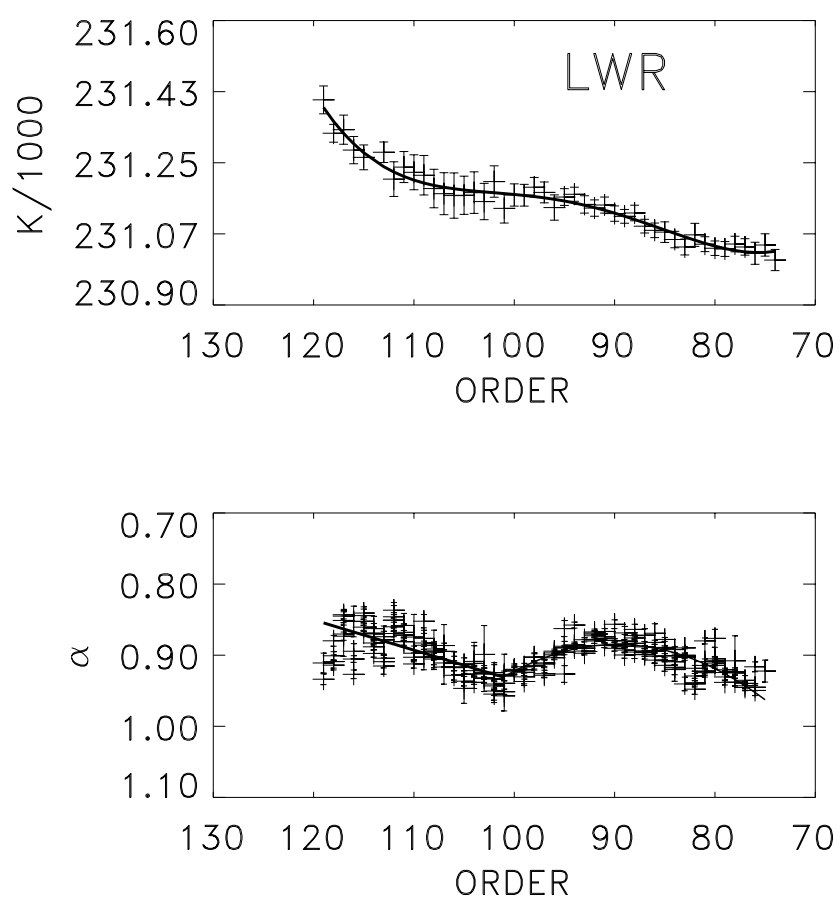

Fig. 6. The $K$ and $\alpha$ parameters for the LWR camera as a function of order number. The least-square polynomial fit to the data as from Eq. (13) and Eq. (16), respectively, are also shown

tion is linear at any date and with a very similar slope, the wavelength differences of individual spectra could be averaged together over the orders 110 to 80 , providing a mean wavelength shift $\Delta \lambda=\lambda_{c}-\lambda_{\text {ref }}$, which is plotted as a function of time in Fig. 4. A linear fit to these data provides:

$\lambda_{\mathrm{c}}-\lambda_{\text {ref }}=52.570796-0.0263910 T$

with a standard deviation of $0.10 \AA$.

Dependence of $\alpha$ on order number and focussing conditions: We find that the ripple parameter $\alpha$ shows a strong dependence on the order number. A linear fit to the data provides:

$\alpha=0.406835+0.01077191 m-5.94540610^{-5} m^{2}$

and a standard deviation of 0.00867 . The corresponding uncertainty on fluxes in the overlap region midway between adjacent orders is $1.4 \%$.

We have also investigated to which extent the central wavelengths depend on the camera head temperature THDA. To this purpose we have selected 194 spectra of calibration standards and computed the wavelength difference $\Delta \lambda=\lambda_{\mathrm{c}}-\lambda_{\text {ref }}$ averaged over the order range 110-80. The results indicate that there is no correlation between 

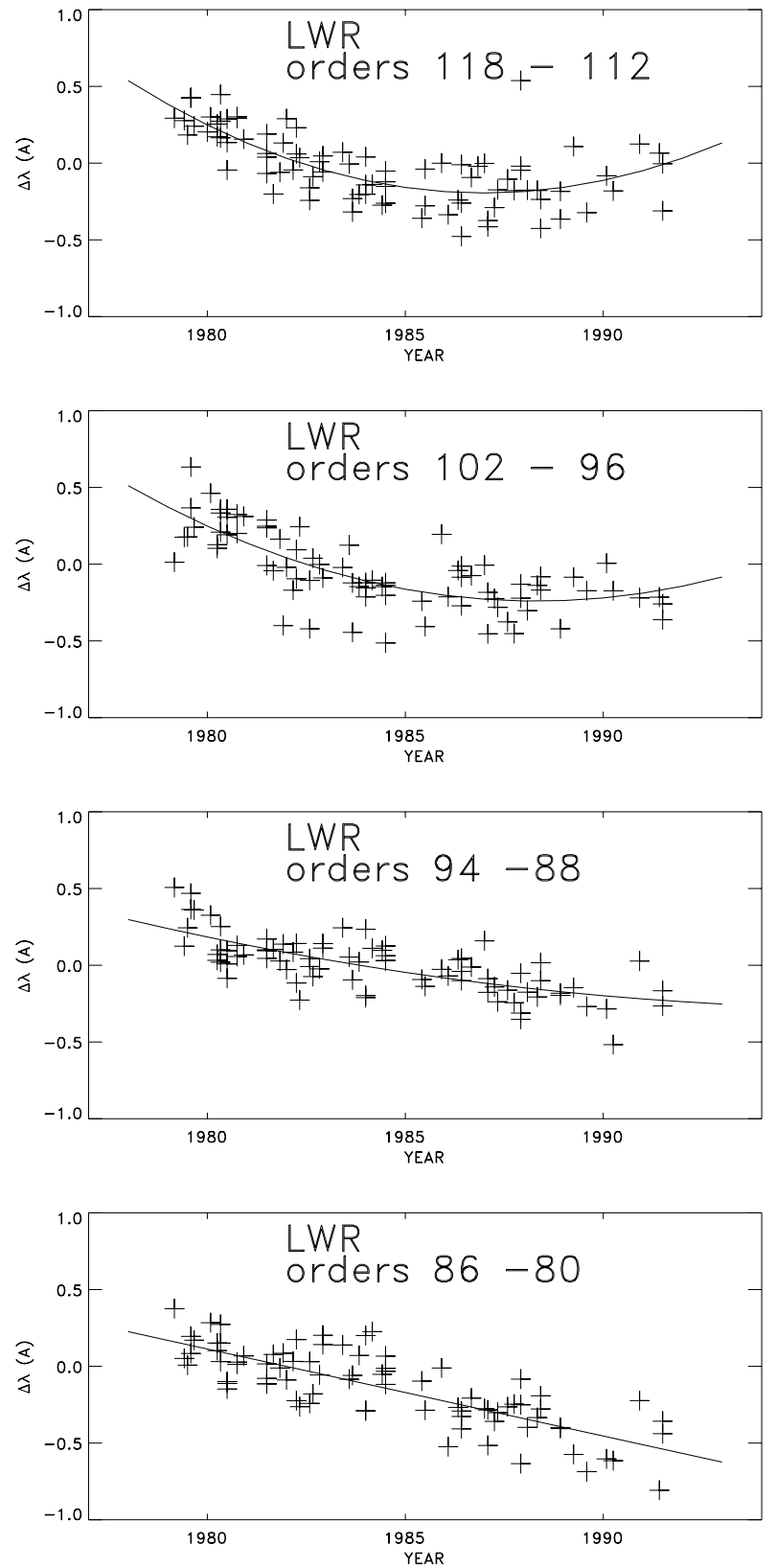

Fig. 7. Examples of how the wavelength shift $\Delta \lambda=\lambda_{\mathrm{c}}-\lambda_{\text {ref }}$ for the LWR camera depends on the time of observations for four different ranges of orders. The coefficients of the least-square polynomial fit to the data are given in Table 1

$\Delta \lambda$ and THDA, unlike the case of the SWP camera, as shown in Fig. 4.

Similarly, no correlation has been found between the $\alpha$ parameter and the focussing conditions, nor between the blaze parameters and the exposure time or the energy distribution of the star. The main results for the LWP camera are provided in Fig. 4 , which shows the $K$ parameter as a function of order number, the wavelength shift $\Delta \lambda=\lambda_{\mathrm{c}}-\lambda_{\text {ref }}$ as a function of observing date (fractional year) and THDA, and the $\alpha$ parameter as a function of order number and focus STEP.
We find that, in spite of the strong dependence of the central positions of spectral orders on the time of observations (see Eq. 11), the quality of the ripple correction remains good even for spectra obtained several years apart, as shown in the example of Fig. 5.

\subsubsection{LWR}

Dependence of $K$ on order number: As for the LWP camera, the central wavelengths of LWR spectral orders $\lambda_{c}(m)$ depend strongly on the date of the observations, but are insensitive to THDA variations.

Following the same procedure used for LWP spectra, we have in first place determined a mean curve $K(m)=$ $m \lambda_{\mathrm{c}}(m)$ for a fixed epoch, to be taken as a reference. A restricted sample of 58 spectra of IUE calibration standards obtained between 1982 and 1986 was used for this purpose. The mean observing date was $T=1982.6$ and the mean values of THDA and focus STEP were $13.40 \pm 1.70^{\circ} \mathrm{C}$ and $-1.3 \pm 0.9$, respectively. The central wavelengths of spectral orders were then averaged together to obtain a mean value of $K=m \lambda_{\mathrm{c}}(m, T=1982.6)$. A fourth order polynomial fit to the results provides:

$K=A+B m+C m^{2}+D m^{3}+E m^{4}$

where

$A=0.28174963510^{6}$

$B=-0.22356558510^{4}$

$C=0.36531948210^{2}$

$D=-0.262477775$

$E=0.70146405510^{-3}$.

The standard deviation of the fit (18.06) corresponds to an uncertainty on the central wavelengths of $0.18 \AA$ at order 100 .

We find that the central wavelengths of LWR spectral orders $\lambda_{\mathrm{c}}(m)$ do not show any dependence on the camera head temperature THDA, as for the LWP camera.

Dependence of $\lambda_{\mathrm{c}}$ on time: The central wavelengths obtained through Eq. (13) are strictly valid only for the $T=1982.6$ reference date. To study the dependence of the central wavelengths on time we have used 86 LWR spectra of IUE calibration standards obtained between 1978 and 1994, and namely: 17 spectra of HD 3360, 12 spectra of HD 34816 and 19 spectra of HD 120315. The results indicate that, differently from the LWP camera, the dependence on observing time is generally non-linear, and it varies across the camera faceplate. We have computed the mean value $\Delta \lambda(m)=\lambda_{\mathrm{c}}(m)-\lambda_{\text {ref }}(m)$ in 10 overlapping windows, each containing seven consecutive orders centered at

$m_{i}=115-4(i-1) \quad$ with $i=1,10$. 
Table 1. Coefficients to determine the wavelength shifts for the LWR camera

\begin{tabular}{clll}
\hline Order & $a\left(m_{i}\right)$ & $b\left(m_{i}\right)$ & $c\left(m_{i}\right)$ \\
\hline 115 & 35736.699 & -35.970790 & 0.0090515542 \\
111 & 62433.199 & -62.859885 & 0.0158223297 \\
107 & 53287.133 & -53.638642 & 0.0134980459 \\
103 & 42742.709 & -43.014583 & 0.0108219635 \\
99 & 28040.843 & -28.206111 & 0.0070930274 \\
95 & 10463.439 & -10.501169 & 0.0026347077 \\
91 & 6223.1919 & -6.2320255 & 0.0015601476 \\
87 & 4478.2512 & -4.4662128 & 0.0011134034 \\
83 & 112.32014 & -0.00566704 & 0 \\
79 & 156.16074 & -0.0787367 & 0 \\
\hline
\end{tabular}

The averages were made from order $m_{i}-3$ to order $m_{i}+3$. A quadratic fit to the data is appropriate in all cases except near the long wavelength end of the camera, where a linear behaviour is found. In summary, the wavelength shift $\Delta \lambda=\lambda_{\mathrm{c}}-\lambda_{\text {ref }}$ can be represented as:

$\Delta \lambda\left(m_{i}, T\right)=a\left(m_{i}\right)+b\left(m_{i}\right) T+c\left(m_{i}\right) T^{2}$

where $T$ is the date of observation (fractional year). The coefficients applicable to individual orders are obtained by linear interpolation of the data in Table 1 . For orders greater than 115 and smaller than 79 the coefficients for $m=115$ and $m=79$ should be used, respectively.

Dependence of $\alpha$ on order number and focussing conditions: As in the case of the LWP camera, we find that the ripple parameter $\alpha$ has a strong dependence on the order number. A polynomial fit to the data provides:

$$
\begin{aligned}
\alpha= & 1.360633-4.25262610^{-3} m \\
& (m=119-101) \\
\alpha= & 3.757863-0.0640201 m+3.566439010^{-4} m^{2} \\
& (m=100-74)
\end{aligned}
$$

with rms errors of 0.02 and 0.01 , respectively.

No significant correlation was found between the $\alpha$ parameter and the focussing conditions.

In Fig. 6 we show $K$ and $\alpha$ as a function of order number for the mean date 1991.2, and in Fig. 7 the mean wavelength shift $\Delta \lambda=\lambda_{\mathrm{c}}-\lambda_{\text {ref }}$ as a function of observing date.

It is interesting to note that, in spite of the strong time dependence of the central positions of spectral orders (see Eq. 15), the LWR ripple correction algorithm provides good results even if applied to spectra obtained several years apart, as shown in Fig. 8.

\subsubsection{The ripple correction algorithm}

In conclusion, the blaze function for $I U E$ high resolution spectra processed with NEWSIPS remains analytically

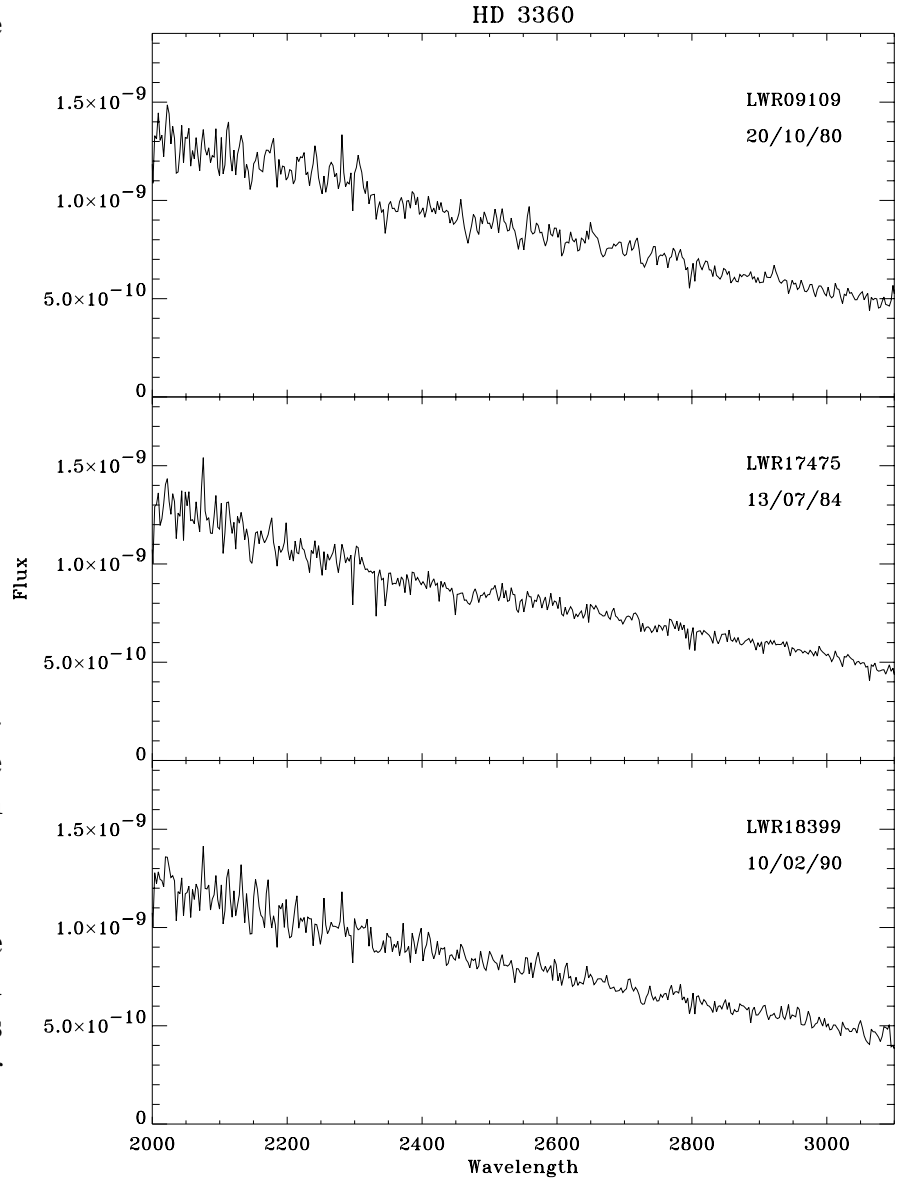

Fig. 8. Flux distribution of HD 3360 obtained from three LWR ripple corrected and flux calibrated high resolution rebinned spectra obtained several years apart. Fluxes are in the same units as in Fig. 3

defined by Eq. (1), where the $\alpha$ parameter is given in Eqs. (9), (12) and (16) for SWP, LWP and LWR, respectively, and the central wavelengths are computed according to:

SWP:

$\lambda_{\mathrm{c}}(m)=137508.316 / m+0.0321729$ THDA $+2.111841(17)$

which is the result of combining Eqs. (7) and (8).

LWP:

$\lambda_{\mathrm{c}}(m)=230868.1770 / m-0.0263910 T+56.433405$

which is the result of combining Eqs. (10) and (11).

LWR:

$\lambda_{\mathrm{c}}(m)=K(m) / m+\Delta \lambda(m, T)$

where $K$ is given by Eq. (13) and $\Delta \lambda(m, T)$ is computed according to Eq. (15). The ripple correction algorithms 


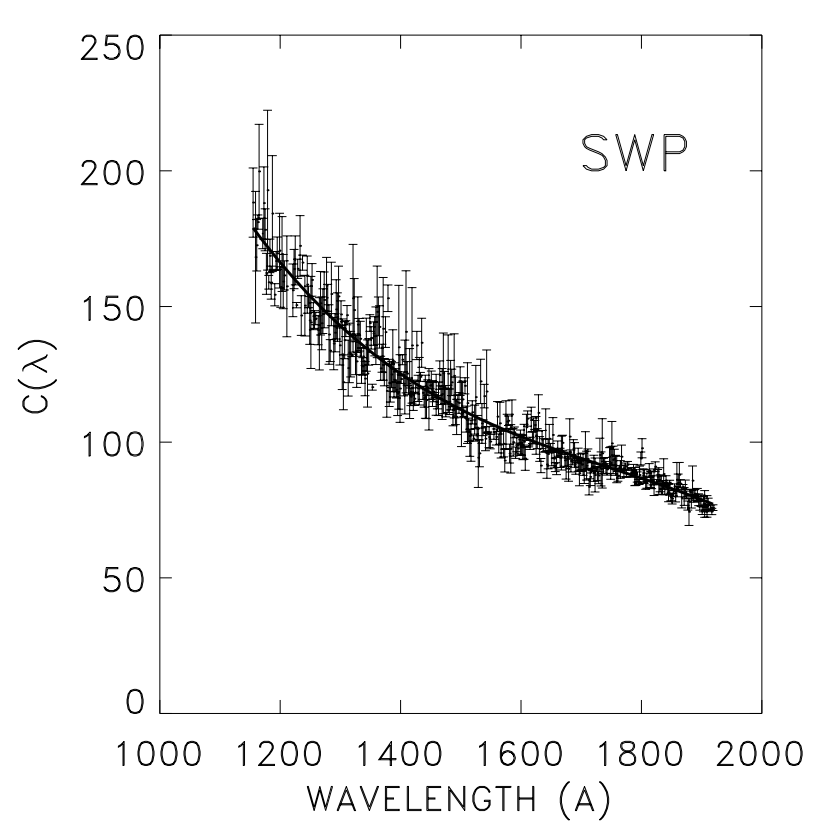

Fig. 9. The high resolution calibration function $C(\lambda)$ for SWP spectra. Error bars are indicated. The thick line represents the polynomial representation in Eq. (22)

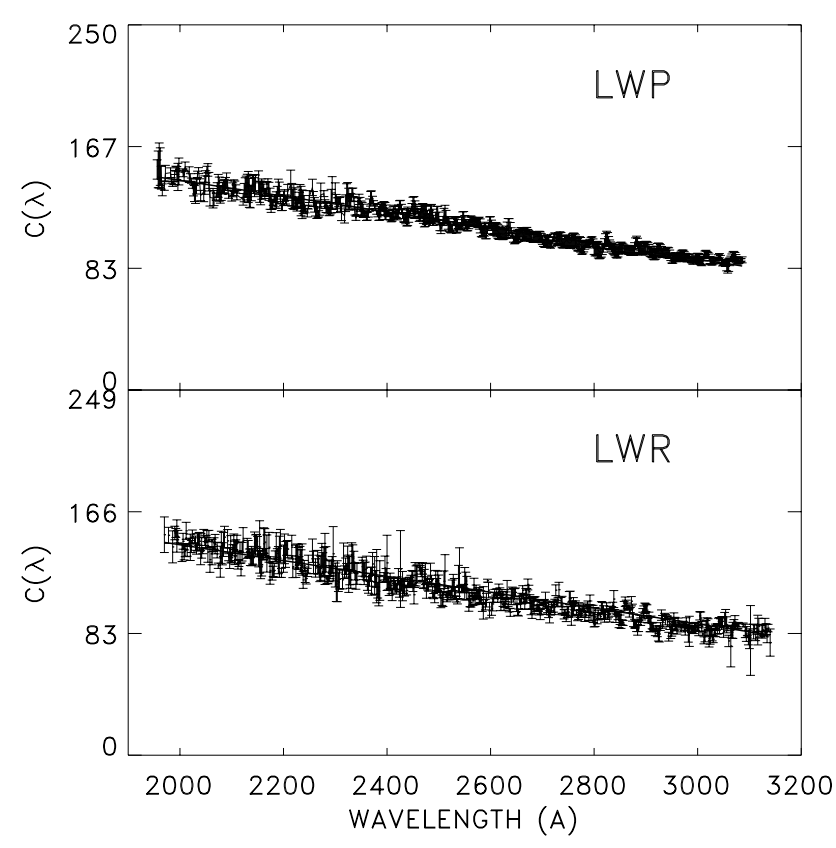

Fig. 10. The high resolution calibration function $C(\lambda)$ for LWP and LWR spectra. Error bars are indicated. The thick line represents the adopted linear representation of $C(\lambda)$ in Eq. (23), which is the same for the LWP and LWR cameras

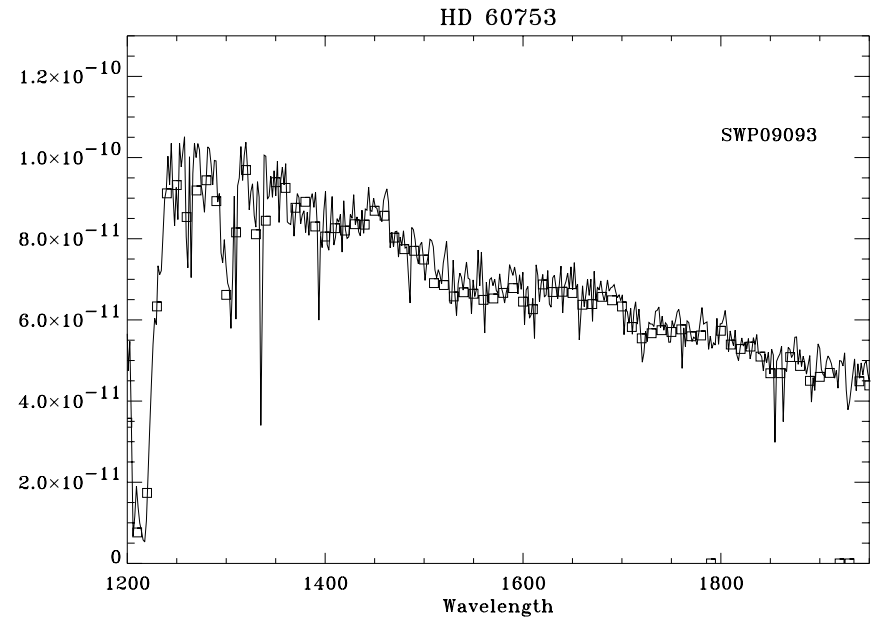

Fig. 11. The flux distribution of the IUE standard HD 60753 obtained from the high resolution spectrum SWP 9093 (full line) is compared with the absolute fluxes of the same star (open boxes). The high resolution spectrum has been rebinned to the low resolution wavelength step. Fluxes are in the same units as in Fig. 3

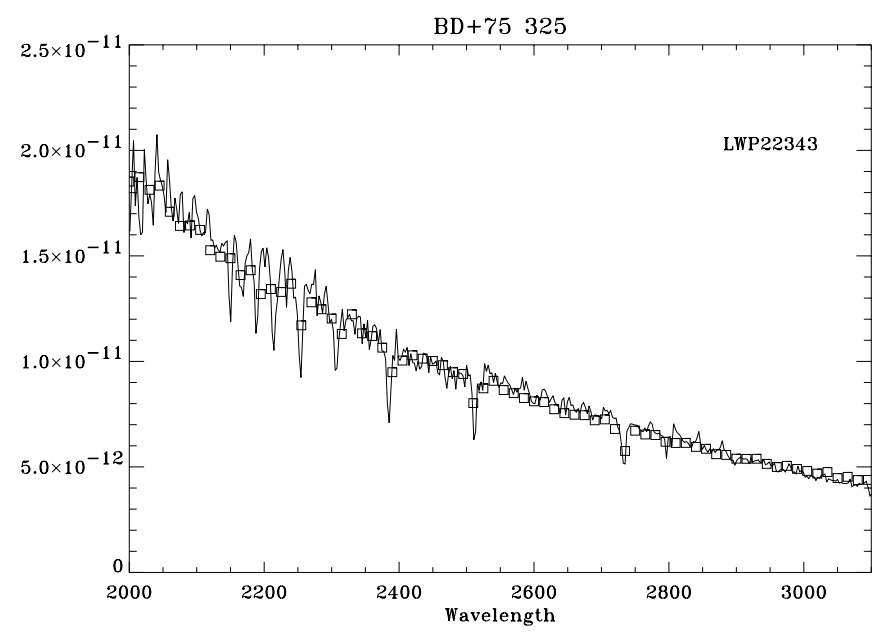

Fig. 12. The flux distribution of the IUE standard BD +75325 obtained from the high resolution spectrum LWP22343 (full line) is compared with the absolute fluxes of the same star (open boxes). The high resolution spectrum as been rebinned to the low resolution wavelength step. Fluxes are in the same units as in Fig. 3

were derived using spectral orders 119 to 72 for the SWP camera, 118 to 74 for the LWP camera and 118 to 76 for the LWR camera. Extrapolation of the algorithms to higher and lower orders provides in general satisfactory results.

\section{The absolute calibration of NEWSIPS high resolution spectra}

For the absolute flux calibration of NEWSIPS high resolution spectra we have followed the method described in 


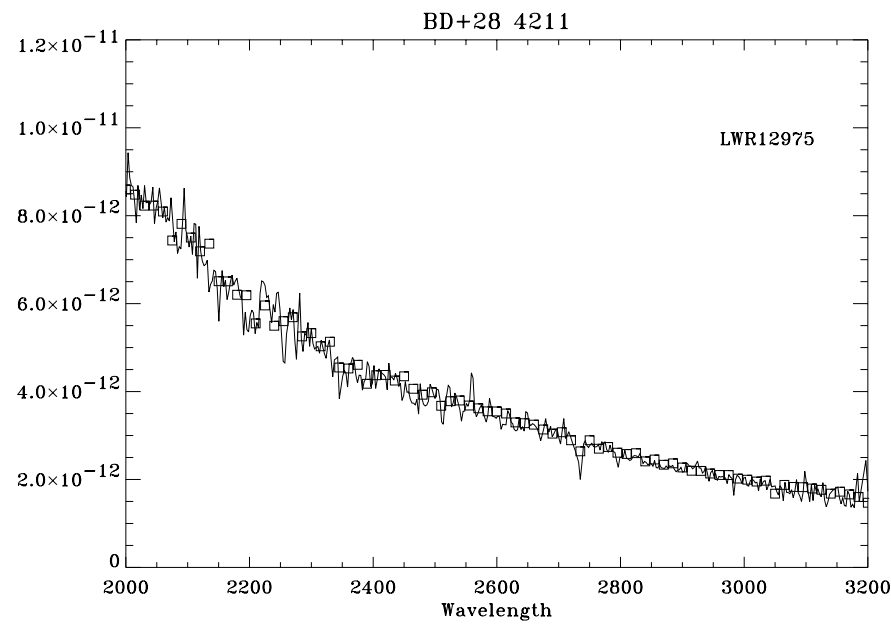

Fig. 13. The flux distribution of the $I U E$ standard BD+28 4211 obtained from the high resolution spectrum LWR12975 (full line) is compared with the absolute fluxes of the same star (open boxes). The high resolution spectrum has been rebinned to the low resolution wavelength step. Fluxes are in the same units as in Fig. 3

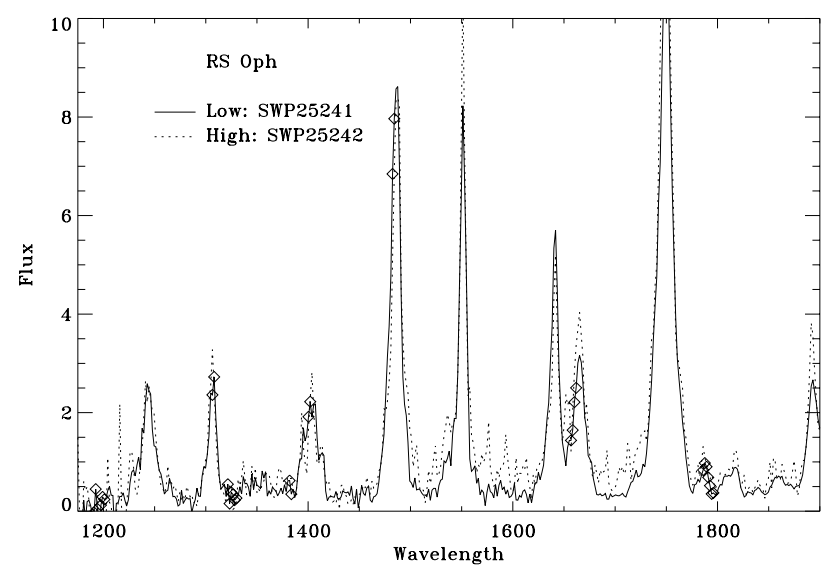

Fig. 14. Comparison between the flux calibrated high and low resolution spectra of the recurrent nova RS Oph. Diamonds mark flagged pixels (saturated or affected by reseau marks) in the low resolution spectrum. Fluxes are in units of $10^{-12} \mathrm{erg}$ $\mathrm{cm}^{-2} \mathrm{~s}^{-1} \AA^{-1}$

Cassatella et al. (1994). Let us indicate with $N(\lambda)$ the ripple-corrected high resolution Flux Numbers, normalized to the exposure time. The corresponding absolute flux can be determined from

$F(\lambda)=C(\lambda) S^{-1}(\lambda) N(\lambda) \operatorname{erg} \mathrm{cm}^{-2} \mathrm{~s}^{-1} \AA^{-1}$

where $S^{-1}(\lambda)$ is the low resolution inverse sensitivity function appropriate to the camera considered, and $C(\lambda)$ is the so-called high resolution calibration function defined as

$C(\lambda)=n(\lambda) / N(\lambda)$

being $n(\lambda)$ the net Flux Numbers (i.e., not absolutely calibrated), normalized to the exposure time, derived from

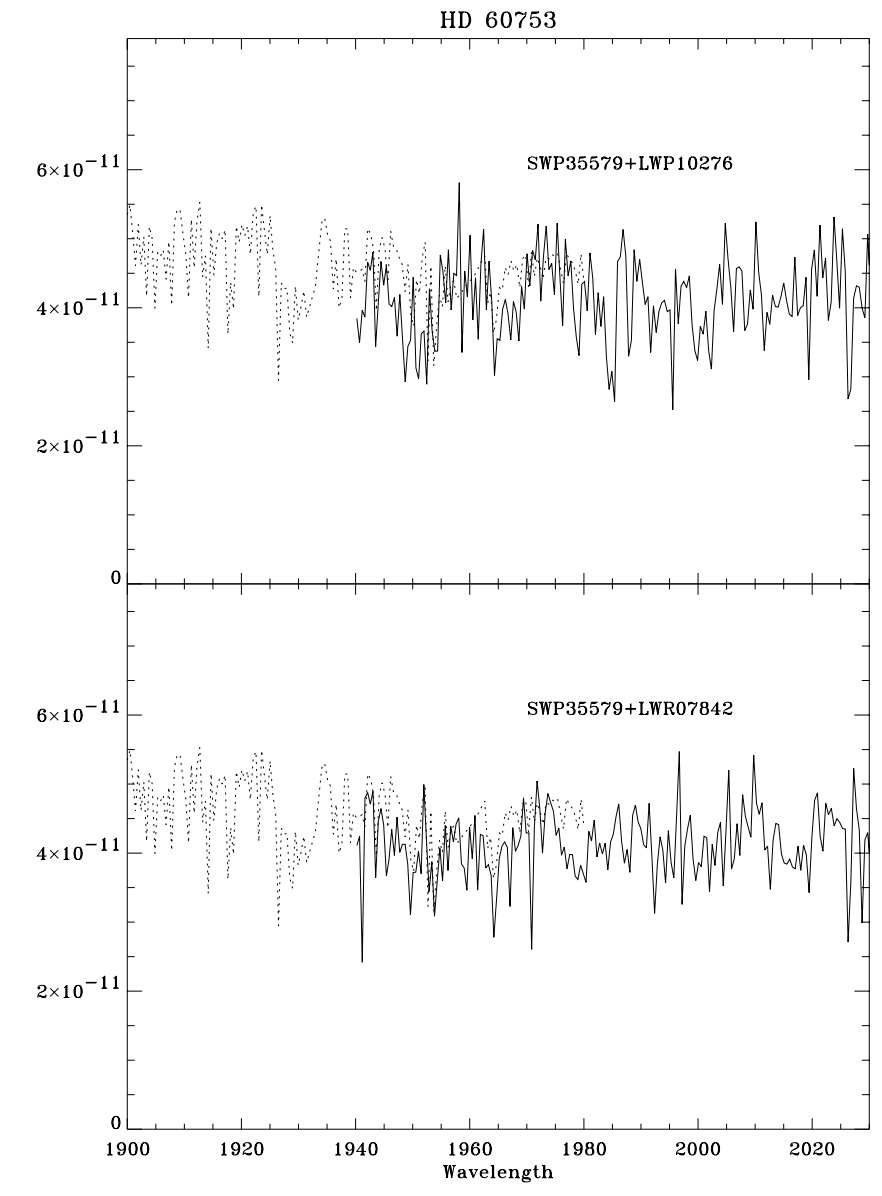

Fig. 15. The combined SWP-LWP and SWP-LWR spectra of HD 60753 are shown in the overlap region around $1950 \mathrm{~A}$. The original data have been rebinned in $0.5 \AA$ steps. Fluxes are in the same units as in Fig. 3

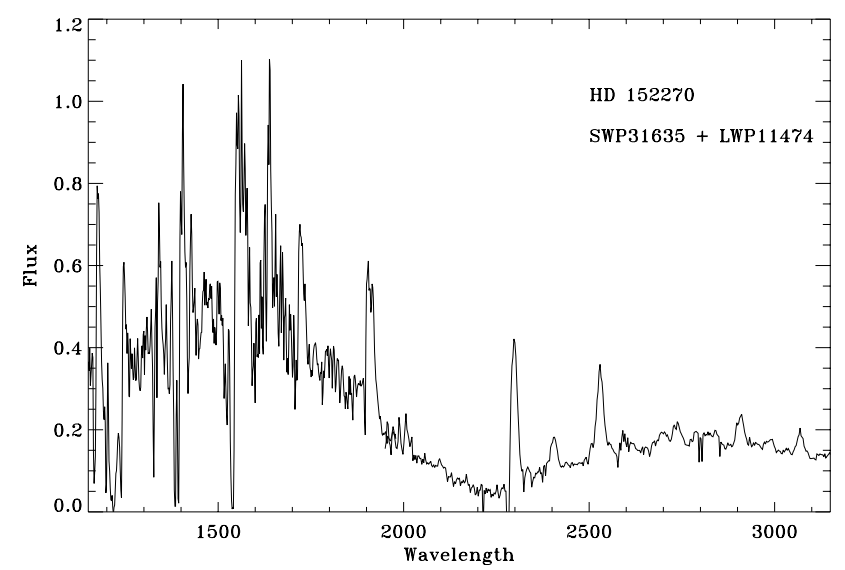

Fig. 16. Combined SWP-LWP spectrum of the Wolf Rayet star HD 152270 after application of the ripple correction and absolute calibration algorithms here described. The high resolution spectra have been resampled to the low resolution wavelength step for display purposes. Fluxes are in units of $10^{-12} \mathrm{erg} \mathrm{cm}^{-2}$ $\mathrm{s}^{-1} \AA^{-1}$ 
low resolution observations of the same target. Because of the time-dependent sensitivity degradation of the cameras, the pairs of low-high resolution spectra used to determine $C(\lambda)$ should be obtained close enough in time. Alternatively, both $n(\lambda)$ and $N(\lambda)$ should be previously corrected for sensitivity degradation. This latter approach, here followed, has the advantage of increasing considerably the number of usable spectra. The correction for sensitivity degradation of high resolution spectra has been made with the same algorithms used for low resolution spectra, as described by Garhart $(1992,1993)$ and Garhart et al. (1997). This procedure is justified in Paper III, which shows that high resolution spectra obtained even several years apart, once corrected in this way, provide very nearly the same flux repeatability performance as spectra obtained close in time.

The high resolution spectra are first corrected for the blaze function, resampled in $2 \AA$ bins, and normalized to the exposure time in seconds. The spectra are then corrected for sensitivity degradation, for the THDA induced sensitivity variations, and for the camera rise time following the same algorithms used in the NEWSIPS processing of low resolution spectra (see González-Riestra et al. 1999b, Paper IV). Finally, the spectra of each target are averaged together to obtain a mean spectrum $N(\lambda)$. To obtain $C(\lambda)$, the mean high resolution spectra of a given target are then divided by the mean low resolution spectrum of the same target (which are also corrected for temperature effects, camera rise-time and sensitivity degradation).

\section{1. $S W P$}

To determine the high resolution calibration function $C(\lambda)$ we have used 28 SWP high resolution spectra of $\mathrm{BD}+28$ 4211, 38 of BD+75 325, 27 of HD 60753,6 of G191 B2B and 13 spectra of CD-38 10980. We find that the repeatability errors on $N(\lambda)$ (after all the above mentioned corrections are applied) are typically $3-5 \%$. These small errors confirm the validity of applying the low dispersion degradation rates to high dispersion spectra.

The low resolution net fluxes $n(\lambda)$ of the above targets were obtained by averaging many low resolution spectra obtained during the $1990-1991$ re-calibration period. The curve $C(\lambda)$ for the SWP camera is shown in Fig. 9.

A third order polynomial fit to the data provides:

$C(\lambda)=A+B \lambda+C \lambda^{2}+D \lambda^{3}$

where

$$
\begin{aligned}
& A=1349.8538 \\
& B=-2.0078566 \\
& C=1.1025258510^{-3} \\
& D=-2.093932710^{-7}
\end{aligned}
$$

with an standard deviation of 6.3 . The above equation has been derived from data in the wavelength range 1175 to $1950 \AA$.

The repeatability error on $C(\lambda)$ is $4 \%$, irrespective of wavelength, which we take as the internal error of the high resolution calibration function.

\section{2. $L W P$}

To determine $C(\lambda)$ for the LWP camera we have used 25 high resolution spectra of $\mathrm{BD}+284211,37$ of $\mathrm{BD}+75325$, 27 of HD 60753 and 4 spectra of CD-38 10980. The repeatability errors on $N(\lambda)$ reach the $4 \%$ level at $2400 \AA$, but do not exceed $2-3 \%$ around $2800 \AA$. Similarly to the case of the SWP camera, these small errors confirm the applicability of the low dispersion sensitivity degradation algorithm to high resolution data. The low resolution net fluxes $n(\lambda)$ of the above targets were obtained by averaging many low resolution spectra obtained during the 19901991 re-calibration period, extracted with NEWSIPS and corrected for time-dependent sensitivity degradation according to Garhart (1993). The curve $C(\lambda)$ for the LWP camera is shown in Fig. 10. A linear fit to the measurements provides:

$C(\lambda)=251.383956-0.053935103 \lambda$

with a standard deviation of 3.49 . The repeatability of the $C(\lambda)$ function is about $4 \%$. The above equation has been derived from data in the wavelength range 1975 to $3150 \AA$ A.

\section{3. $L W R$}

To determine $C(\lambda)$ we have used a total of 17 high and 23 low resolution spectra of the calibration standards BD+28 4211, BD+75 325, HD 60753 and HD 93521.

We find that the repeatability errors on the net fluxes $N(\lambda)$ after the sensitivity degradation correction are of the same order as for the LWP camera, confirming once more the applicability of the low dispersion sensitivity degradation algorithm to high resolution spectra.

The resulting determinations of $C(\lambda)$ are reported in the bottom panel of Fig. 10. It is interesting to note that the data points are well fitted by the same analytical representation as for the LWP camera (Eq. 23). The residuals correspond to an rms error on $C(\lambda)$ of 5.3. A linear fit would provide about the same residuals (4.5), and the curve would only deviate from that of the LWP by $1.3 \%$. The internal accuracy of the calibration function ranges from $5 \%$ below $2300 \AA$ to $3 \%$ at longer wavelengths. The wavelength range covered by the LWR high resolution absolute calibration is the same as for the LWP camera.

Note that also Cassatella et al. (1994) found that the $C(\lambda)$ curve is the same for LWP and LWR data processed with IUESIPS. 


\subsection{Examples of application}

It is important to compare the fluxes obtained from high resolution spectra using the present method with the fluxes of the calibration standards which define the IUE flux scale (Paper IV). Examples of such a comparison are given in Figs. 11, 12 and 13 referring to the stars HD 60753, BD+28 4211 and BD+75 325 observed with the SWP, LWP and LWR cameras, respectively.

In the above examples, the agreement between high and low resolution fluxes is within the $4 \%$ repeatability errors quoted above.

It should be stressed that the present calibration is applicable to both continuum and emission line sources. This is confirmed by line emission measurements in several pairs of low-high resolution spectra of emission line sources. As an example, we show in Fig. 14 a low and a high resolution spectrum of the recurrent nova RS Oph taken very close in time.

Another test made was to verify the accuracy of the absolute calibration in the overlap region around $1950 \AA$ between the SWP and the LWP and LWR cameras. We find that, in this region, the short and long wavelength cameras agree to within 2 to $6 \%$ on average. As an example, we show in Fig. 15 the overlap region for two pairs of spectra of HD 60753.

The good match between short and long wavelength high resolution spectra can also be deduced from Fig. 16, which shows a combined SWP-LWP spectrum of the Wolf Rayet star HD 152270.

We note that residual non-linearity effects in the Intensity Transfer Function, especially in the case of underexposed spectra, or spectra near the saturation limit, can occasionally cause a flux mismatch between the short and long wavelength cameras.

\section{Conclusions}

We have shown that a reliable correction for the echelle blaze function can be obtained for IUE high resolution spectra processed with NEWSIPS. We find that not only the $K$ parameter, but also the $\alpha$ parameter varies with order number, and that the central peaks of the blaze function deviate from the reference positions in a way which depends on the value of the THDA temperature for the SWP camera, and on the observation date for the LWP and LWR cameras. Having taken into account all these dependences (and not only the dependence of $K$ on order number as in previous investigations), allows the present algorithm to successfully correct most spectra. According to our experience, the algorithm might fail in a few cases (probably less then $5-10 \%$ ). The identified cases of failure correspond to images in which the interorder background level is not properly evaluated with the NEWSIPS automatic extraction procedure or to cases in which the target is not acquired (purposely or not) in the center of the large entrance apertures of the $I U E$ spectrographs. In this respect, we note that a displacement of 1 arcsec along the dispersion line corresponds to a velocity shift of the wavelength scale of about $5 \mathrm{~km} \mathrm{~s}^{-1}$, irrespective of the camera used. For example, a displacement of 5 arcsec, or $0.125 \AA$ at $1500 \AA$ would produce a flux mismatch in the overlap region midway between adjacent orders of about $4 \%$.

As for the absolute calibration of IUE high resolution spectra, we have shown that its internal accuracy is about $4 \%$ and that it applies both to continuum and emission line sources. The flatter shape of the $C(\lambda)$ function found here is due to the improved background extraction procedure in NEWSIPS (Smith 1999).

Acknowledgements. We would like to acknowledge the contribution of all VILSPA staff to the development and production of the INES system and the referee, Dr. Joy S. Nichols, for useful comments.

\section{References}

Ahmad I.A., 1981, IUE NASA Newslett. 14, 129

Ake T., 1981, IUE NASA Newslett. 15, 60

Cassatella A., Selvelli P.L., Ponz J.D., González-Riestra R., Vogel M., 1994, A\&A 281, 594

Garhart M.P., 1992, IUE NASA Newslett. 48, 98

Garhart M.P., 1993, IUE NASA Newslett. 52, 27

Garhart M.P., Smith M.A., Levay K.L., Thompson R.W., 1997, IUE NEWSIPS Information Manual, Version 2.0

González-Riestra R., Cassatella A., Solano E., Altamore A., Wamsteker W., 1999a, A\&AS (in press) (Paper III)

González-Riestra R., et al., 1999b (in preparation) (Paper IV) Grady C.A., Garhart M.P., 1989, IUE NASA Newslett. 27, 102 Nichols J.S., Linsky J.L., 1996, AJ 111, 517

Rodríguez-Pascual P.M., González-Riestra R., Schartel N., Wamsteker W., 1999, A\&AS 139, 183 (Paper I)

Smith M.A., 1999, PASP 760, 722

Wamsteker W., Skillen I., Ponz J.D., de la Fuente A., Barylak M., Yurrita I., 1999, Ap\&SS (in press) 